Meta

Journal des tradlucteurs

Translators' Journal

\title{
L'esprit de famille synonymique
}

\section{Robert Dubuc}

Volume 13, numéro 3, septembre 1968

URI : https://id.erudit.org/iderudit/002643ar

DOI : https://doi.org/10.7202/002643ar

Aller au sommaire du numéro

Éditeur(s)

Les Presses de l'Université de Montréal

ISSN

0026-0452 (imprimé)

1492-1421 (numérique)

Découvrir la revue

Citer cet article

Dubuc, R. (1968). L'esprit de famille synonymique. Meta, 13(3), 132-133.

https://doi.org/10.7202/002643ar d'utilisation que vous pouvez consulter en ligne.

https://apropos.erudit.org/fr/usagers/politique-dutilisation/ 


\section{L'ESPRIT DE FAMILLE SYNONYMIQUE}

« Il revenait à Bunuel de nous donner à sentir le néant du cinéma mexicain et à travers son Ange exterminateur, si révélateur lors d'une seconde vision de fustiger les mœurs d'une grande bourgeoisie. ${ }^{1}$ Ces lignes d'un jeune critique de cinéma, ne nous intéressent ni par le «néant du cinéma mexicain » ni par les «mœurs d'une grande bourgeoisie », mais plutôt par l'emploi du mot vision dans un sens qui ne manquera pas de faire sourciller les délicats. Cet usage n'est pourtant pas tout à fait nouveau. Jean Giraud ${ }^{2}$ en a relevé un exemple qui remonte à 1919.

Quel enrichissement cette acception de vision apporte-t-elle à notre vocabulaire ? Il semble bien qu'on ait senti, d'abord au cinéma et ensuite à la télévision, le besoin d'un mot pour désigner l'action intelligente d'un spectateur qui regarde un spectacle au grand ou au petit écran. On avait déjà des mots de sens voisins, mais aucun ne rendait adéquatement l'idée à exprimer.

Il y a d'abord représentation (representation). Représentation est un terme de théâtre. On représente une pièce, c'est-à-dire on la joue. On assiste à une représentation, mais il peut y avoir, à la rigueur, représentation sans spectateurs c'est évidemment éphémère ! À la télévision, comme au cinéma, on dit de préférence présentation. Exemple: «La présentation de Square à Jeudi-Théâtre n'a pas rallié les suffrages de la critique. »Dans le secteur qui nous occupe, cinéma et télévision, présentation désigne encore une manifestation au cours de laquelle on présente à la presse, aux critiques, etc., un film ou une série télévisée. Exemple: Il y aura présentation de la série d'Iberville à l'occasion d'un coquetel auquel vous êtes cordialement invité. Présentation se traduit souvent en ce sens par preview ${ }^{3}$.

Représentation et présentation se trouvent du même côté de la barricade: ces mots désignent une action du producteur, du cinéaste, du propriétaire de salle, mais non du spectateur. Ils ne peuvent donc se substituer à vision.

De son côté, projection (projection) désigne une opération technique par laquelle on projette un film sur un écran à l'aide d'un appareil de projection ou projecteur (projector). C'est donc une opération matérielle qui n'a rien à voir directement avec la réalité qu'on veut désigner.

Il faut arriver à visionnement pour trouver un terme dont le champ sémantique soit très voisin de celui de vision. Mais les deux termes ne sont pas de purs synonymes. Le visionnement (screening) ne se fait pas pour le plaisir: c'est d'ordinaire un travail auquel se livrent censeurs, critiques, montcurs ou réalisateurs. On visionne un film soit à des fins techniques (montage, postsynchronisation, synchro-

1. Louis Marcorelles, Nouvelles littéraires, 28 juin 1962.

2. Jean Giraud, le Lexique français du cinéma des origines à 1930, Paris, Centre national de la recherche scientifique, 1958, p. 205.

3. Ibid., p. 164. 
nisation, doublage, etc.), soit à des fins d'appréciation (pour juger de la valeur morale ou esthétique d'un film).

Si l'on veut indiquer l'action «honnête » de regarder un film ou une émission pour son plaisir, il reste donc vision (viewing).

Ce tour d'horizon serait incomplet si l'on n'ajoutait pas à cette famille synonymique quelques vagues cousins qui ne sont pas sans intérêt.

Sous les régimes dits de restrictions, il faut souvent amortir le coût de certaines productions de prestige en les "réchauffant», c'est-à-dire en les servant une seconde ou une troisième fois au public. On désigne ces présentations multiples par le mot diffusion (pass ${ }^{4}$ ) et le numéro d'ordre pertinent. Exemples: «Samedi, en soirée, la seconde diffusion de Cyrano de Bergerac, qui fut, à sa création à la télévision, un succès. ${ }^{5}$ "Certains programmes font l'objet d'une seconde diffusion. " 6

La seconde diffusion se distingue, en télévision, de la reprise. La seconde diffusion implique le passage intégral de l'émission initiale; la reprise suppose une nouvelle réalisation, mais avec le même scénario. Ainsi quand on passe le cinégramme (kinescope recording) d'une émission qui a déjà été programmée, on fait une seconde diffusion et non une reprise. Par contre, quand Radio-Canada a repris, à quelques années d'intervalle, le ballet l'Enfant et les sortilèges, il a fait une reprise, parce qu'il s'agissait d'une nouvelle production: la distribution (casting) n'était pas tout à fait la même et la mise en scène non plus; seul le scénario était identique. Faudrait-il alors conclure que reprise serait le terme fançais à substituer à l'anglicisme remake ${ }^{\top}$ ? Rien n'est jamais aussi simple. La reprise utilise le même scénario; le remake se contente de traiter le même sujet. Cette distinction, toutefois, semble précaire. Peut-être aurait-on profit à la laisser tomber. On pourrait alors donner à reprise un sens assez étendu pour l'appliquer également à la réalité désignée sous le nom de remake.

ROBERT DUBUC

4. On trouve aussi dans le jargon des gens du métier le mot passage. * Il faut prévoir au moins trois passages pour ce film. " Cet emploi est peut-être un anglicisme, mais il n'est pas circonscrit au Québec. On trouve ce mot dans les comptes rendus des délibérations de la Communauté des télévisions francophones.

5. Paul Vialar, les Nouvelles littéraires, 12 juillet 1962.

6. Jean Quéval et Jean Thévenot, TV, Paris, Gallimard, 1957, p. 20.

7. «A ce mot anglais passé dans le vocabulaire courant du cinéma on donne souvent en France le sens de sous-produit. Cette interprétation n'est nullement celle du jeune critique Louis Marcorelles; il considère, tout au contraire, que la reprise périodique des mêmes sujets est un phénomène qui conditionne dans une large mesure la production cinématographique. » Les Nouvelles littéraires, 22 février 1962. 